\title{
Shikonin blocks migration and invasion of human breast cancer cells through inhibition of matrix metalloproteinase-9 activation
}

\author{
SOON YOUNG JANG ${ }^{1 *}$, JAE KOAN LEE ${ }^{1 *}$, EUN HYANG JANG ${ }^{1}$, SEO YOUNG JEONG ${ }^{2}$ and JONG-HO KIM ${ }^{1}$ \\ ${ }^{1}$ College of Pharmacy, ${ }^{2}$ Department of Life and Nanopharmaceutical Science, \\ Kyung Hee University, Seoul 130-701, Republic of Korea
}

Received February 26, 2014; Accepted April 2, 2014

DOI: $10.3892 /$ or.2014.3159

\begin{abstract}
Shikonin, a natural naphthoquinone isolated from a traditional Chinese medicinal herb, has been reported to promote tumor cell death. However, there are few reports concerning its effect on metastasis-related cell invasion and migration behavior. In the present study, we investigated the effect of shikonin on human breast cancer invasion and migration. We found that shikonin inhibited phorbol 12-myristate 13-acetate (PMA)-induced cell migration and invasion in MCF-7 breast cancer cells, which was correlated with modulation of matrix metalloproteinase-9 (MMP-9) through suppression of both expression and proteolytic and promoter activity. We also found that shikonin inhibited both MMP-9 expression and promoter activity in MDA-MB-231 cells with high metastatic potential. These results indicated that shikonin induces the suppression of migration and invasion through modulation of MMP-9 in human breast cancer cells. Therefore, shikonin may be a potential anticancer drug for human breast cancer therapy.
\end{abstract}

\section{Introduction}

Breast cancer ranks among the most common malignant tumors afflicting women worldwide (1). Cancer metastasis is the leading cause of mortality in patients with breast cancer; breast cancer survival rates fall from $90 \%$ for localized to $20 \%$ for metastatic disease. Therefore, controlling metastasis

Correspondence to: Professor Seo Young Jeong, Department of Life and Nanopharmaceutical Science, Kyung Hee University, 1 Hoegidong, Dongdaemun-gu, Seoul 130-701, Republic of Korea

E-mail: syjeong@khu.ac.kr

Professor Jong-Ho Kim, College of Pharmacy, Kyung Hee University, 1 Hoegi-dong, Dongdaemun-gu, Seoul 130-701, Republic of Korea E-mail: jonghokim@khu.ac.kr

*Contributed equally

Key words: shikonin, cell invasion, cell migration, matrix metalloproteinase-9, human breast cancer cells and invasion represents an important therapeutic strategy (2). Metastasis requires that invasive cells detach from localized tumors by degrading the extracellular matrix using proteases, including matrix metalloproteinases (MMPs); survive in the circulation as circulating tumor cells (CTCs); and colonize distant locations. Thus, understanding the molecular mechanisms underlying each of these steps is essential for targeting metastatic cells at an early stage and improving patient survival (3).

MMPs are a family of extracellular matrix (ECM)degrading enzymes comprising 24 members. Based on their substrates, MMPs are divided into four subclasses: collagenase, gelatinase, stromelysin and membrane-associated (4). As a main ECM-degrading enzyme family, MMPs have essential roles in physiologic processes such as tissue development, remodeling and wound healing (5). However, they are also involved in certain tissue destructive diseases, such as atherosclerosis, inflammation, rheumatoid arthritis, and tumor invasion, metastasis and neoangiogenesis (6). Recent studies have shown that MMPs are important elements of the tumor microenvironment, regulating tumor progression, metastatic niche formation and inflammation in cancer (7). Among human MMPs, MMP-2 (gelatinase-A) and MMP-9 (gelatinase-B) are key enzymes in the degradation of type IV collagen, which is an important component of the ECM. MMP-2 and MMP-9 are the MMP members primarily associated with tumor migration, invasion and metastasis of various types of cancers (8). It has been shown that enhanced expression of MMP-9 is associated with the progression and invasion of tumors, whereas MMP-2 is usually expressed constitutively $(9,10)$. Thus, researchers have focused on discovering inhibitors of MMP activity as a strategy for suppressing tumorigenesis and metastasis.

Shikonin is an active naphthoquinone compound and the main component of red pigment extracts from the Chinese medicinal herb, Lithospermum erythrorhizon Sieb et Zucc. Shikonin and its analogues can kill cancer cells through a number of mechanisms, including inhibition of topoisomerase-I (11), polo-like kinase 1 (PLK1) and protein tyrosine kinase (PTK) (12); regulation of phosphorylation-dependent activities of extracellular-regulated protein kinase (pERK), c-Jun $\mathrm{N}$-terminal kinase (JNK) and protein kinase Ca (PKCa) (13); suppression of tumor necrosis factor receptor-associated protein 1 (TRAP1) expression (14); activation of caspases (15); 
and inhibition of proteasome activity (16). In previous studies, shikonin and its derivatives were shown to exert antiproliferative and pro-apoptotic effects against a number of tumor cells, including sarcoma 180 (S-180) ascites cells, gastric cancer, colon adenocarcinoma and oral cancer (17). A recent report also showed that shikonin activates the p53 and caspase-9 pathways (18) in human malignant melanoma A375-S2 cells. However, little is known concerning its anti-invasion effects in human breast cancer cell lines. In the present study, we investigated the anti-invasion effects of shikonin and explored the possible molecular mechanisms in breast cancer using the human breast cancer cell lines, MDA-MB-231 and MCF-7. We showed that shikonin inhibits the migration and invasion of breast cancer cells by suppressing AP-1 transcription factormediated MMP-9 expression.

\section{Materials and methods}

Materials and reagents. Shikonin was purchased from Sigma (St. Louis, MO, USA) and antibodies against MMP-2 and MMP-9 were purchased from Calbiochem (Boston, MA, USA). Antibodies against PCNA and p65 were purchased from Santa Cruz Biotechnology Inc. (Santa Cruz, CA, USA). MMP-9 ELISA kits were purchased from R\&D Systems (Abingdon, UK).

Cell line and cell culture. Human breast cancer cell lines, MCF-7 and MDA-MB-231, were used in the present study. Cells were grown in RPMI-1640 medium supplemented with $10 \%$ fetal bovine serum (FBS).

Transwell invasion assays. The invasion of tumor cells was assessed in Transwell chambers. A Transwell membrane $(8-\mu \mathrm{m}$ pore size, 6.5-mm diameter; Corning Costar Corporation) was used. A Transwell membrane coated with Matrigel $(100 \mu \mathrm{g} / \mathrm{ml}$, $100 \mu \mathrm{l} /$ well) was used for the invasion assay. Cells were seeded onto the upper wells in the presence of different concentrations of shikonin. The bottom chambers of the Transwell were filled with conditioned medium. After incubation for $24 \mathrm{~h}$, cells were fixed, stained and counted under a light microscope.

In-gel zymography. The MMP activities were assayed as previously described (19). Briefly, $5 \times 10^{5}$ cells in a 6 -well plate were seeded and maintained in serum-free medium for $16-24 \mathrm{~h}$. The conditioned medium was separated on a sodium dodecyl sulfate-polyacrylamide gel electrophoresis gel containing $1 \mathrm{mg} / \mathrm{ml}$ gelatin. The gel was washed with buffer I [Tris- $\mathrm{HCl}$ (pH 7.5) and 2.5\% Triton X-100)], incubated overnight in buffer II $\left[150 \mathrm{mM} \mathrm{NaCl}, 5 \mathrm{mM} \mathrm{CaCl}{ }_{2}, 50 \mathrm{mM}\right.$ Tris-HCl (pH 7.6)] at $37^{\circ} \mathrm{C}$ and stained with Coomassie blue. The clear bands indicate where MMPs degraded gelatin.

Cell migration assay. Migration was assessed by a wound healing assay. Cells were seeded at $2 \times 10^{4} \mathrm{MCF}-7$ and MDA-MB-231 cells/well. After scraping the cell monolayer with a sterile micropipette tip, the wells were washed with serum-free medium several times with different concentrations of shikonin. The first image of each scratch was acquired at time zero. At $24 \mathrm{~h}$, each scratch was examined and captured at the same location and the healed area was measured.
Western blotting. Cells were washed twice with $1 \mathrm{X}$ PBS, and cell extracts were prepared using RIPA buffer (1X PBS, $1 \%$ NP-40, $0.5 \%$ sodium deoxycholate, $0.1 \%$ SDS containing an additional $100 \mathrm{ml}$ of $10 \mathrm{mg} / \mathrm{ml} \mathrm{PMSF}$, and 1 Complete Mini Protease Inhibitor tablet). Lysate proteins were resolved by SDS-PAGE and transferred using nitrocellulose membranes (Whatman Protran BA83; $0.2 \mu \mathrm{m}$ ) The membranes were incubated with TBS buffer containing $0.1 \%$ Tween-20 and 5\% skim milk, and then exposed to the desired primary antibody. After treatment with the proper secondary antibody, the immunoreactive bands were visualized using standard ECL (Pierce, Rockford, IL, USA) method.

Reverse transcription-PCR. In the reverse transcription-PCR (RT-PCR) analysis, total RNA was extracted from the treated cells. For reverse transcription reaction, cDNA was synthesized from $1 \mu \mathrm{g}$ of total RNA using the High Capacity cDNA synthesis kit (Applied Biosystems). The PCR primers used were as follows: MMP-9 sense, 5'-TTTGACAGCGACAAGA AGTGG-3' and MMP-9 antisense, 5'-TCCCATCCTTGAAC AAATACA-3'; MMP-2 sense, 5'-CATTCCGCTTCCAGGG CACAT-3' and MMP-2 antisense, 5'-GCTCCTGAATGCCCT TGAGTCA-3'; GAPDH sense, 5'-GCCATCGTCACCAAC TGGGAC-3' and GAPDH antisense, 5'-CGATTTCCCGCT CGGCCGTGG-3'. PCR products were analyzed by agarose gel electrophoresis and visualized by treatment with ethidium bromide.

Electrophoretic mobility shift assay (EMSA). The oligonucleotides were labeled with $\left[\gamma_{-}{ }^{32} \mathrm{P}\right]$ ATP and incubated with nuclear extracts for $30 \mathrm{~min}$ by using the Gel Shift Assay kit (Promega Corp., Madison, WI, USA). The DNA-protein complexes were resolved on non-denaturing and non-reducing $6 \%$ acrylamide gels. The probes used for EMSA were as follows. Probe for AP-1, 5'-CGC TTG ATG ACT CAG CCG GAA-3'.

Statistical analysis. The results are presented as mean $\pm \mathrm{SE}$, and statistical comparisons between groups were carried out using one-way ANOVA followed by the Student's t-test. $\mathrm{P} \leq 0.05$ was considered to indicate a statistically significant result.

\section{Results}

Effects of shikonin on the migration and invasion of breast cancer cells. To investigate the effects of shikonin on human breast cancer cell migration and invasion, we treated MDA-MB-231 cells with different concentrations of shikonin (1-5 $\mu \mathrm{M})$ and performed wound healing and Matrigel-based Transwell invasion assays. As shown in Fig. 1, shikonin significantly inhibited MDA-MB-231 cancer cell migration by $\sim 80 \%$ and invasion by $\sim 60 \%$. Moreover, shikonin induced a concentration-dependent inhibition of PMA-induced migration and invasion of MCF-7 cells. MCF-7 cells treated with PMA presented a 1.5-fold increase in migration (Fig. 1A and $\mathrm{B}$ ). However, treatment with $5 \mu \mathrm{M}$ shikonin inhibited PMA-induced migration by $\sim 70 \%$ in the MCF-7 cancer cell line (Fig. 1A and B). We next examined whether shikonin inhibits PMA-induced invasion in MCF-7 cells. PMA induced a 2-fold increase in the invasion of MCF-7 cells, while shikonin inhibited this invasion by $\sim 60 \%$ at a $5 \mu \mathrm{M}$ dose 
A
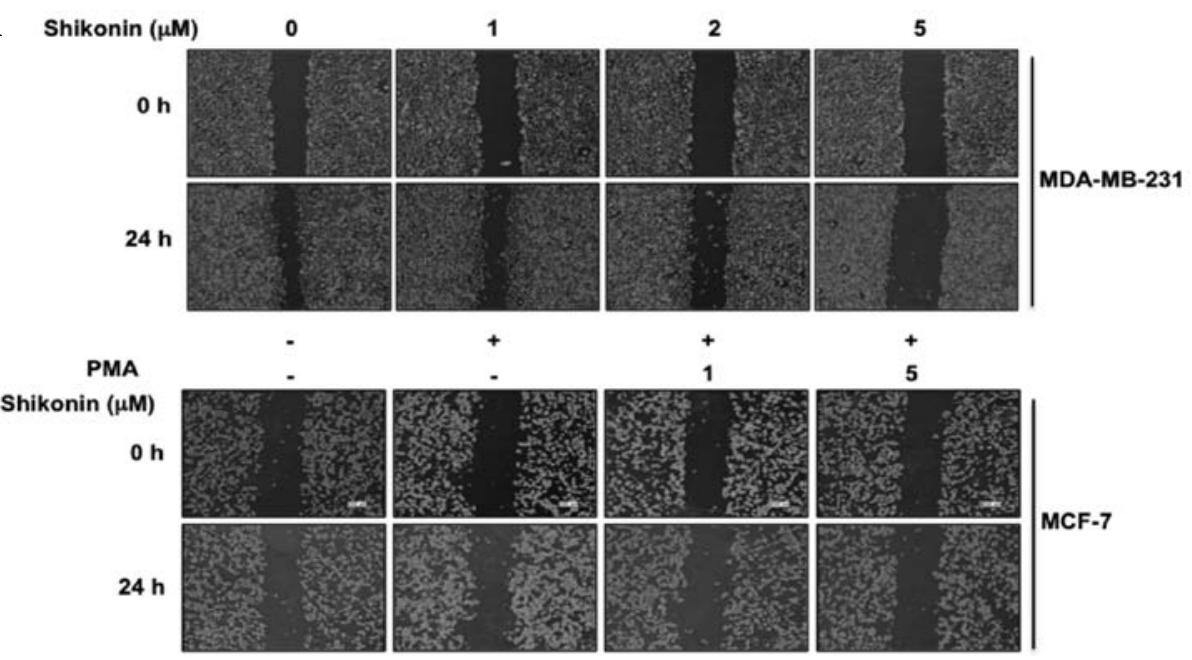

$+$

B
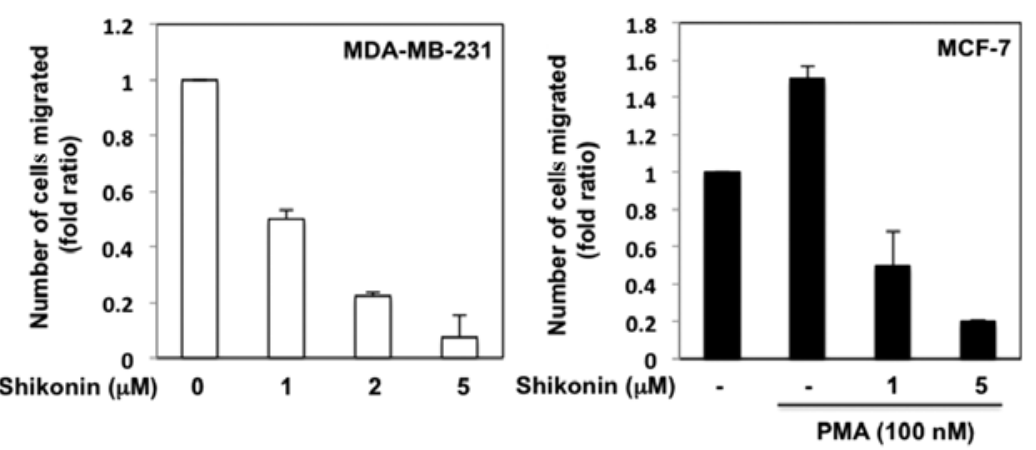

C
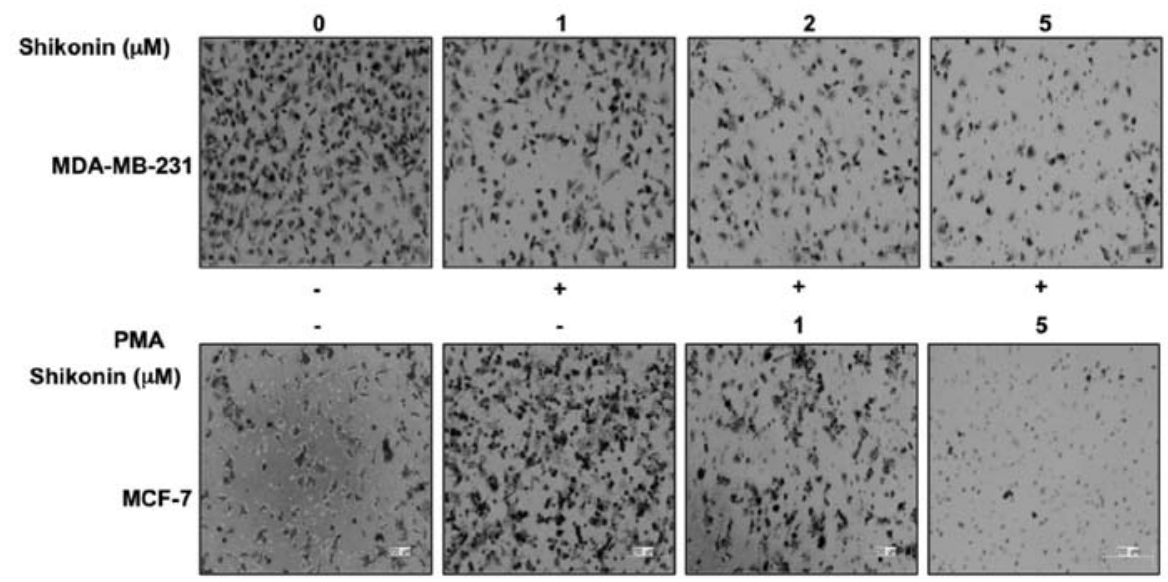

D

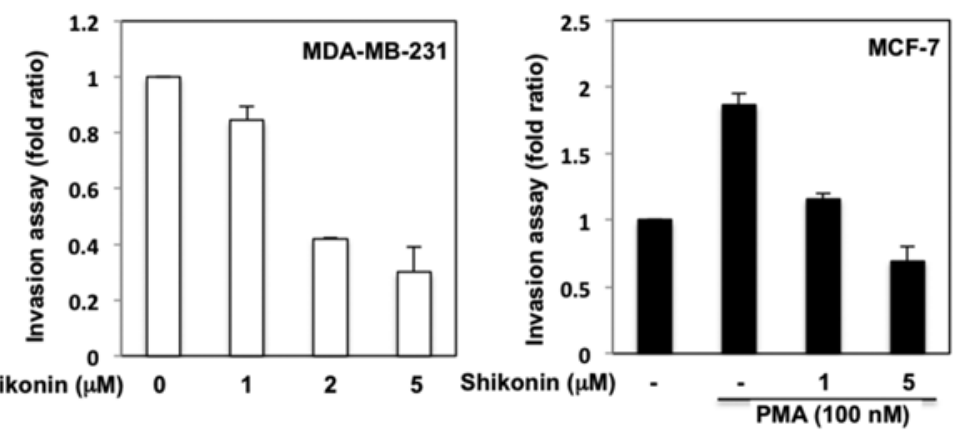

Figure 1. Effects of Shikonin on the migration and invasion of human breast cancer cells. (A) MDA-MB-231 and MCF-7 cells were 'wounded' by scratching with a pipette tip and then were incubated with or without $100 \mathrm{nM}$ PMA and the indicated concentrations of shikonin. Representative images of wound healing were captured at the time of the scratch and $24 \mathrm{~h}$ later. Scale bars, $100 \mu \mathrm{m}$. (B) Cell migration into the wounded area was quantified as the fold ratio of wound healing ( $\mathrm{n}=3) .{ }^{*} \mathrm{P}<0.05,{ }^{* *} \mathrm{P}<0.01$ vs. vehicle alone-treated cells. ${ }^{\prime \prime} \mathrm{P}<0.05,{ }^{\# \#} \mathrm{P}<0.01$ vs. PMA alone-treated cells. (C) Cells were treated with the indicated concentrations of shikonin, with or without $100 \mathrm{nM}$ PMA, for $24 \mathrm{~h}$, and invasion was determined by Transwell assays. Representative photomicrographs of membrane-associated cells were stained with eosin. (D) Cell invasion values were quantified ( $n=3)$. ${ }^{~} \mathrm{P}<0.05$ vs. vehicle alone-treated cells. ${ }^{~} \mathrm{P}<0.05$ vs. $\mathrm{PMA}$ alone-treated cells. 
A
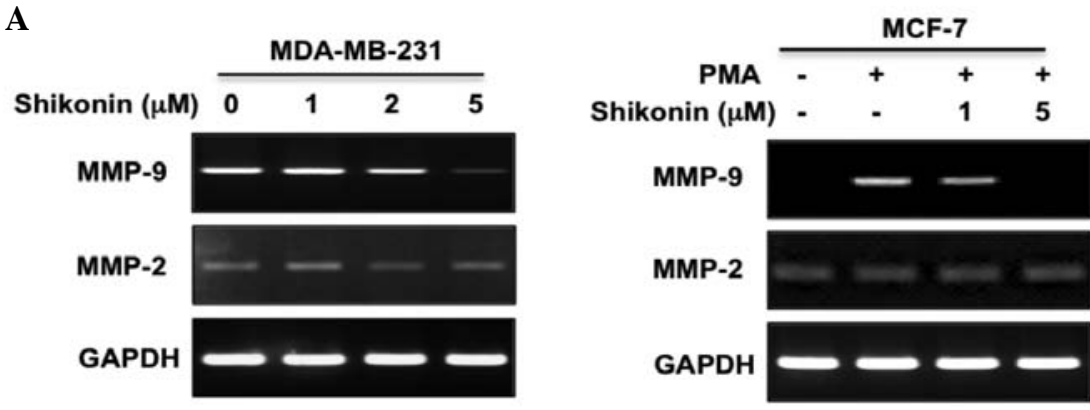

B
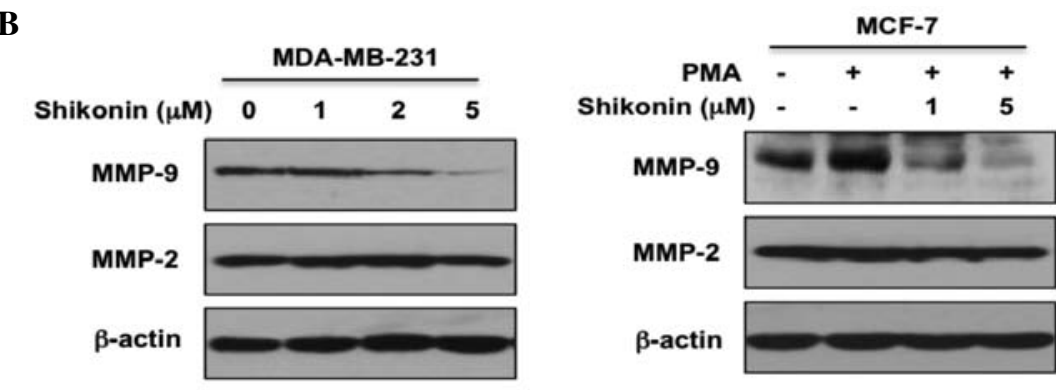

C
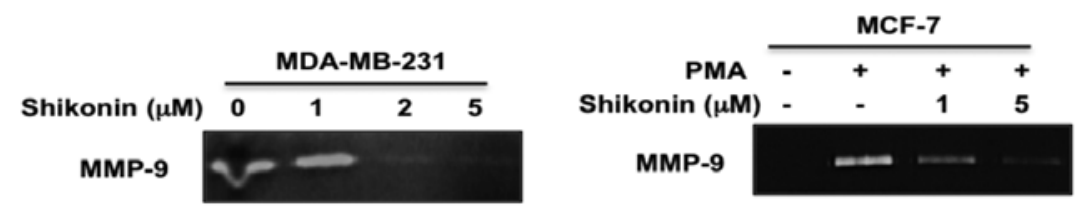

D
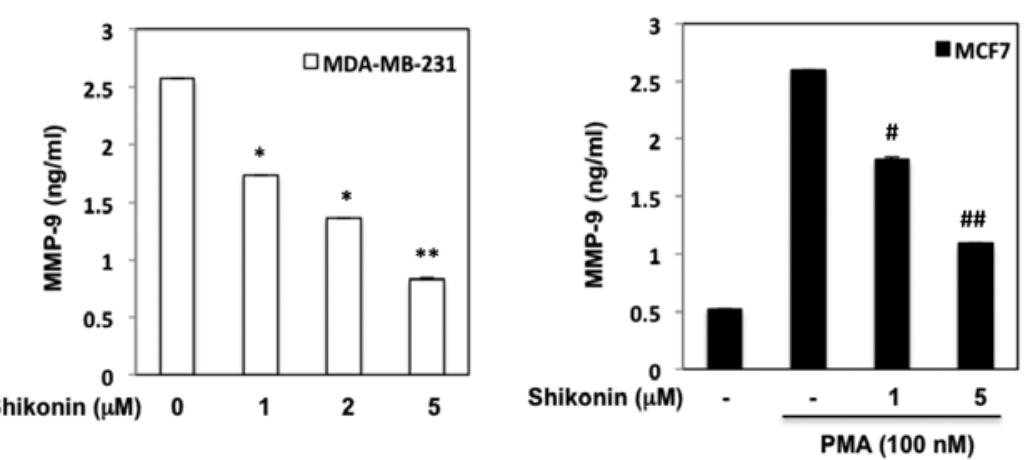

Figure 2. Effects of shikonin on MMP-9 expression and activity in human breast cancer cells. (A) MDA-MB-231 and MCF-7 cells were incubated with the indicated concentrations of shikonin, with or without $100 \mathrm{nM}$ PMA. MMP-9 and MMP-2 mRNA levels were determined by reverse transcription-polymerase chain reaction (RT-PCR). Expression of glyceraldehyde-3-phosphate dehydrogenase (GAPDH) was used as a control. (B) MMP-9 and MMP-2 protein levels in the MDA-MB-231 and MCF-7 breast cancer cells were evaluated by western blot analysis. $\beta$-actin expression was included as an internal control. (C) Cells in serum-free medium were treated with the indicated concentrations of shikonin, with or without $100 \mathrm{nM}$ PMA for $24 \mathrm{~h}$. Cell-conditioned medium was collected after treatment and assayed by gelatin zymography to analyze the activity of MMP-9. Assays were performed several times. (D) Secreted MMP-9 in the conditioned media was quantified by ELISA. ${ }^{*} \mathrm{P}<0.05,{ }^{* *} \mathrm{P}<0.01$ vs. vehicle alone-treated cells. ${ }^{\#} \mathrm{P}<0.05,{ }^{\# \#} \mathrm{P}<0.01$ vs. PMA alone-treated cells. All measurements were performed in triplicate.

(Fig. 1C and D). These data imply that shikonin is an effective inhibitor of breast cancer cell migration and invasion.

Shikonin inhibits MMP-9 expression and proteolytic activity. MMP-9 and MMP-2 are important ECM-degrading enzymes that are reported to be involved in cancer cell invasion and metastasis (20). On the basis of these observations and the foregoing data, we examined the effect of shikonin on the expression of both MMP-2 and MMP-9 in MDA-MB-231 cells; we also investigated PMA-induced MMP-9 expression in MCF-7 cells. Following treatment with shikonin, MMP-9 expression in the MDA-MB-231 cells gradually decreased over time at both the mRNA and protein levels (Fig. 2A and B). Shikonin also significantly inhibited PMA-induced expression of both MMP-9 mRNA and protein in the MCF-7 cells. However, neither PMA nor shikonin treatment affected MMP-2 mRNA or protein expression (Fig. 2A and B). We next examined MMP-2 and MMP-9 secretion and activity using gelatin zymography and enzyme-linked immunosorbent assays (ELISAs), respectively. MMP-9 secretion measured in the MDA-MB-231 cell-conditioned medium was significantly reduced by shikonin (Fig. $2 \mathrm{C}$ and D). Similarly, shikonin 
A

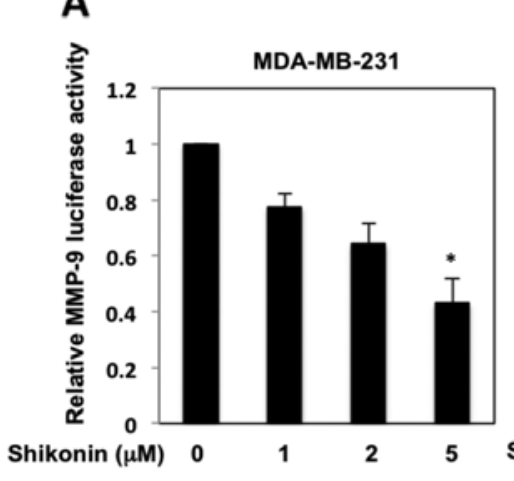

D

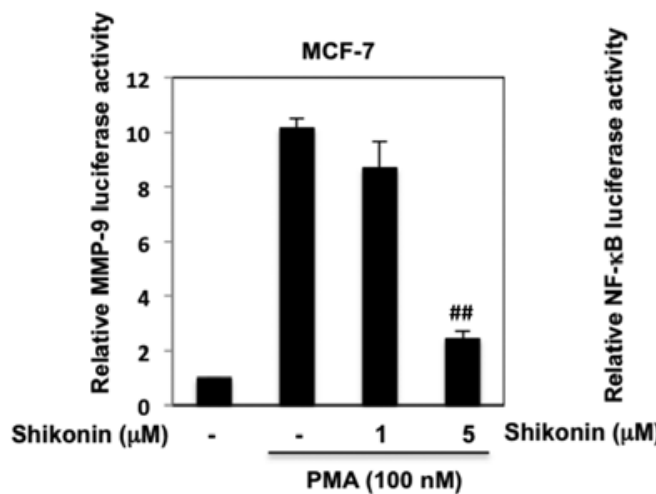

B

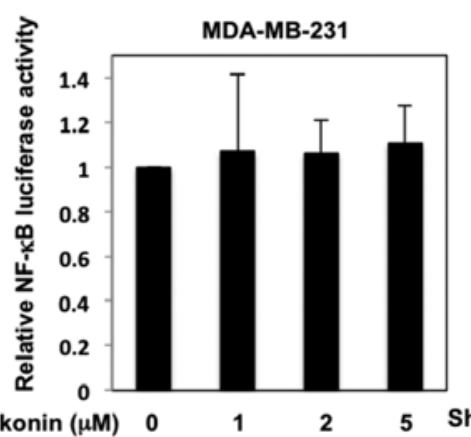

E

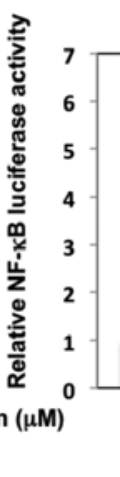

C

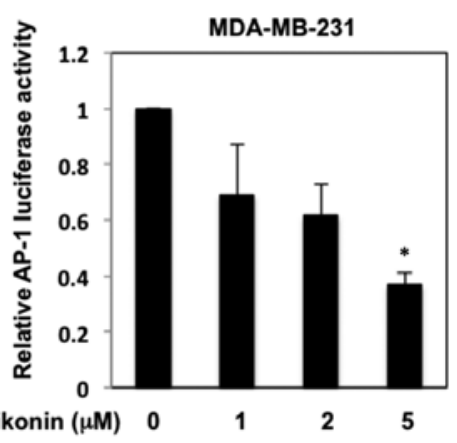

F

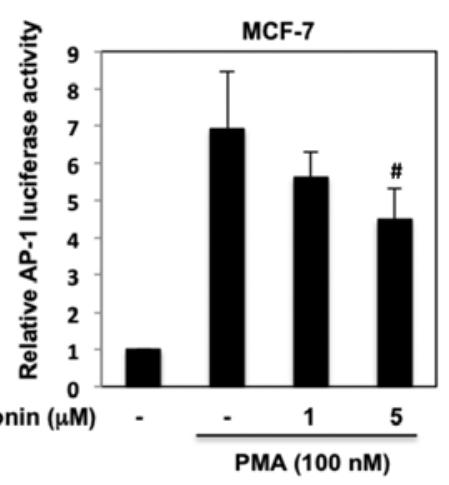

Figure 3. Effect of shikonin on MMP-9 promoter activity. (A and D) Cells were transfected with MMP-9 luciferase reporter plasmid (0.5 $\mu$ g). After 24 h, cells were treated with the indicated concentrations of shikonin for $24 \mathrm{~h}$ in the presence or absence of $100 \mathrm{nM}$ PMA and harvested for luciferase assays. Luciferase activity was normalized to that of $\beta$-galactosidase activity. ${ }^{*} \mathrm{P}<0.05$, vs. vehicle alone-treated cells. ${ }^{\# \#} \mathrm{P}<0.01$ vs. PMA alone-treated cells. (B and $\mathrm{E}$ ) Cells were transfected with the NF- $\mathrm{BB}-\mathrm{Luc}$ vector $(0.5 \mu \mathrm{g})$. The transfected cells were treated with shikonin for $24 \mathrm{~h}$ and incubated with or without $100 \mathrm{nM}$ PMA. Luciferase activity was normalized to that of $\beta$-galactosidase activity. (C and F) Cells were transfected with AP-1 luciferase reporter plasmids $(0.5 \mu \mathrm{g})$. The transfected cells were treated with shikonin for $24 \mathrm{~h}$ and incubated with or without $100 \mathrm{nM}$ PMA and luciferase activities were measured. * $\mathrm{P}<0.05$ vs. vehicle alone-treated cells. ${ }^{\#} \mathrm{P}<0.05$ vs. PMA alone-treated cells. Data are expressed as means \pm SD and are presented as relative luciferase activity. Experiments were performed in triplicate.

inhibited PMA-induced MMP-9 activity in the MCF-7 cells. These results indicate that shikonin is involved in the regulation of proteolytic activity as well as expression of MMP-9.

Shikonin inhibits transcriptional activity of MMP-9 by suppressing AP-1 binding. To explain the molecular mechanism underlying the inhibitory effects of shikonin on MMP-9 expression, we investigated the possible regulation of MMP-9 at the transcriptional level. The MMP-9 promoter contains two AP-1 binding sites and a nuclear factor (NF)- $\kappa \mathrm{B}$ binding site, and it has been shown that NF- $\mathrm{B}$ and AP-1 play critical roles in regulating basal and cytokine-induced MMP-9 expression in many cancer cell lines (19). Therefore, we examined whether shikonin inhibits MMP-9 expression through an effect on NF- $\kappa$ B or AP-1 binding sites in the MMP-9 promoter. Human breast cancer cells, MDA-MB-231 and MCF-7, were transiently transfected with the MMP-9 promoter construct. As shown in Fig. 3A, MMP-9 promoter activity was decreased in a dose-dependent manner following shikonin treatment in MDA-MB-231 cells. We also, confirmed that the MMP-9 promoter was activated up to $\sim 10$-fold in the PMA-treated MCF-7 cells. Shikonin showed a dose-dependent inhibition of PMA-induced MMP-9 promoter activity in the MCF-7 cells (Fig. 3D). Next, we examined whether shikonin affects the transcriptional activity of NF- $\kappa \mathrm{B}$ and AP-1, critical modulators of MMP-9 activity. MDA-MB-231 cells were transiently transfected with AP-1 report vectors and then treatment of shikonin was carried out at different doses. As shown in Fig. 3C, AP-1 promoter activity was significantly reduced in the range of 1-5 mM of shikonin. In addition, MCF-7 cells were transiently transfected with reporter vectors containing tandem repeats of the AP-1 binding site to confirm the specificity of the shikoninmediated inhibitory effect on AP-1. Treatment with PMA led to an 7 -fold increase in AP-1 promoter activity; notably, this stimulatory effect of PMA was significantly reduced by treatment with shikonin (Fig. 3F). However, NF- $\kappa$ B promoter activity exhibited significant change in the MDA-MB-231 and MCF-7 cells (Fig. 3B and E). These results suggest that shikonin inhibits MMP-9 activity through suppression of AP-1-mediated MMP-9 transcription in breast cancer cells.

Shikonin decreases transcription factor binding to the AP-1 motif in the MMP-9 promoter region. Next, we examined whether shikonin inhibits the transcriptional binding activity of AP-1 to its DNA motifs in MDA-MB-231 cells using electrophoretic gel mobility shift assay (EMSA). MDA-MB-231 cells were treated with different dose of shikonin for $24 \mathrm{~h}$ and nuclear extracts were prepared. As shown in Fig. 4A, shikonin significantly decreased AP-1 DNA binding activity. Subsequently, we examined whether the subunit of AP-1 

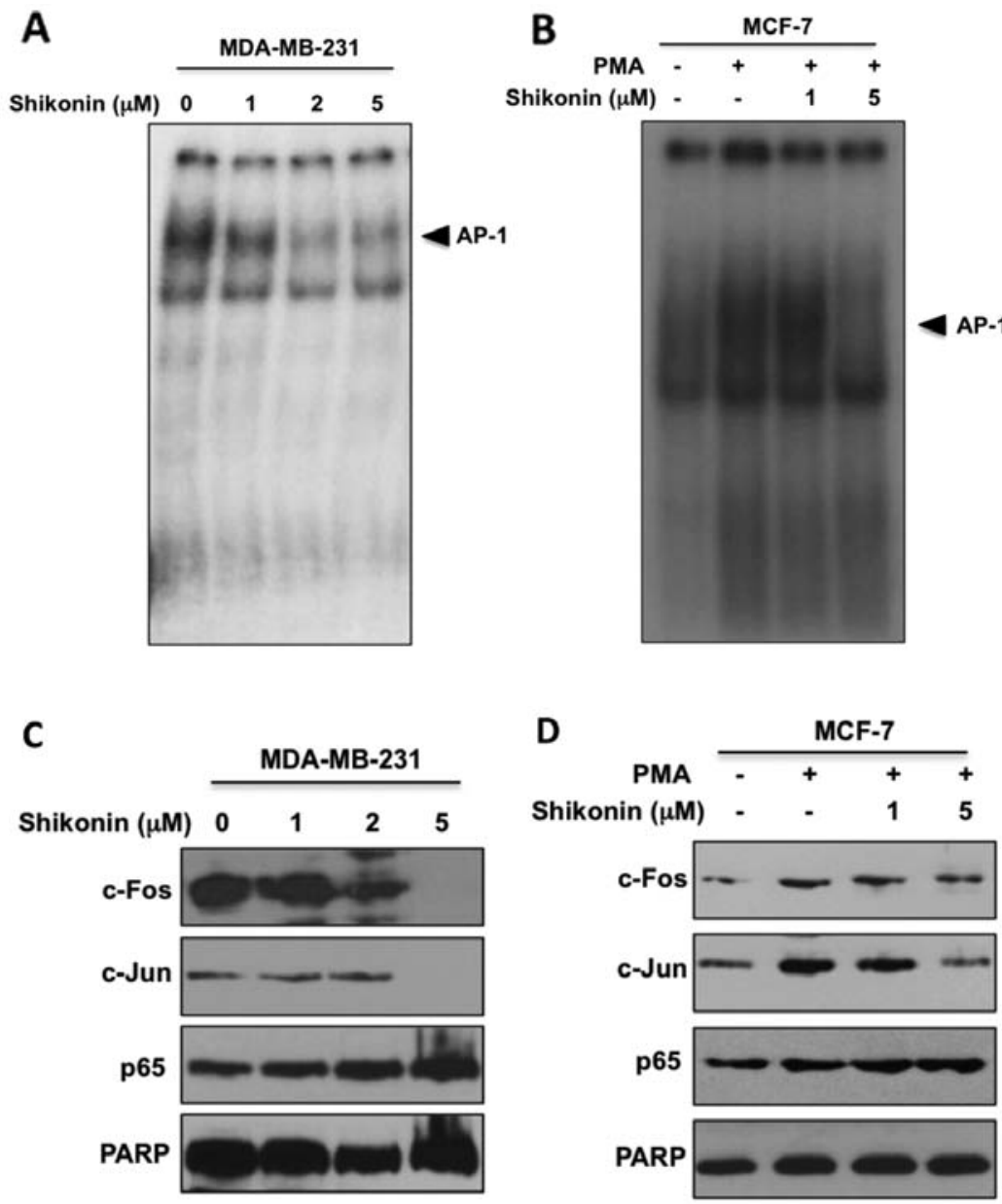

Figure 4. Effects of shikonin on the DNA binding activities and expression of AP-1. (A and B) Cells were treated with shikonin for $24 \mathrm{~h}$ with or without $100 \mathrm{nM}$ PMA, and nuclear extracts $(5 \mu \mathrm{g})$ were prepared. Nuclear extracts were incubated with radiolabeled oligonucleotides containing the AP-1 motif in the MMP-9 promoter. Bound complexes were assayed by electrophoretic gel mobility shift assay (EMSA). (C and D) Cells were treated with shikonin for $24 \mathrm{~h}$ with or without $100 \mathrm{nM}$ PMA, and nuclear extracts were prepared. The nuclear levels of AP-1 (c-Fos and c-Jun) and NF- $\mathrm{kB}$ (p65) subunits were determined by western blot analysis using subunit-specific antibodies. Poly(ADP-ribose) polymerase (PARP) expression was included as an internal control.

transcription factor is regulated by shikonin. Expression levels of c-Fos and c-Jun, subunits of AP-1, were significantly reduced by shikonin treatment (Fig. 4C). Similar results were observed in MCF-7 cells. MCF-7 cells were pretreated with different concentrations of shikonin with $100 \mathrm{nM}$ PMA. Then, nuclear extracts were prepared and analyzed for AP-1 DNA binding activities. As shown in Fig. 4B, shikonin significantly decreased PMA-induced AP-1 DNA binding ability. To determine which subunit of the AP-1 transcription factor is regulated by shikonin, we examined the expression levels of c-Fos and c-Jun following shikonin treatment. Our data showed that shikonin significantly reduced PMA-induced c-Jun and c-Fos expression but had little effect on the expression of p65 in the western blot assays (Fig. 4D). These results suggest that shikonin inhibits AP-1 transcription activity by suppressing its subunit expression.

\section{Discussion}

Members of the MMP family, in particular, MMP-9, are associated with regulation of cancer cell invasion and metastasis, the most insidious and life-threating aspects of cancer $(21,22)$. Therefore, the development of compounds capable of inhibiting MMP-9 could prove invaluable in the treatment of breast cancer patients. Shikonin has previously been shown to possess antiproliferative and pro-apoptotic activity in a number of tumor cells, but there is little information concerning its effects on tumor invasion (23). In the present study, we showed that shikonin effectively inhibited the migration and invasion of human breast cancer cells. We also found that shikonin inhibited the expression of MMP-9 in breast cancer cells, thereby reducing its proteolytic activity. These data suggest that shikonin suppresses the metastatic phenotype of human breast cancer cells via downregulation of MMP-9 expression. Previous studies have reported that the MMP-9 promoter contains a number of transcription factor-binding motifs, including those for NF- $\mathrm{kB}$ and AP-1 (24). The activity of the MMP-9 promoter is induced by AP-1 and/or NF- $\kappa \mathrm{B}$ $(25,26)$, which are two key transcription factors involved in the regulation of MMP-9 gene expression (24). Regulation of these transcription factors is also critically involved in the process of tumor metastasis. AP-1 is composed of members of the c-Jun and c-Fos families (27-29). Upon activation of AP-1, they associate to form a variety of homodimers and heterodimers that localize to the nucleus and regulate gene expression. Our results showed that shikonin decreased nuclear c-Fos 
and c-Jun, without affecting p65. These data suggest that the inhibitory effect of shikonin on AP-1 is responsible for shikonin-induced inhibition of MMP-9 expression/activity, and subsequent suppression of invasion.

In summary, we demonstrated that shikonin inhibits human breast cancer cell migration and invasion by suppressing MMP-9 activity by blocking the activation of AP-1 transcription factors. Therefore, we conclude that shikonin is a potential therapeutic agent that may be valuable in preventing the metastasis of several types of human cancers, including breast cancer.

\section{Acknowledgements}

This research was supported by the grant from Basic Science Research Program through the National Research Foundation of Korea grant funded by the Ministry of Education, Science, and Technology of the Korea government (2011-0010430).

\section{References}

1. Jemal A, Siegel R, Ward E, Hao Y, Xu J and Thun MJ: Cancer statistics, 2009. CA Cancer J Clin 59: 225-249, 2009.

2. Cho HJ, Kang JH, Kwak JY, et al: Ascofuranone suppresses PMA-mediated matrix metalloproteinase-9 gene activation through the Ras/Raf/MEK/ERK- and Ap1-dependent mechanisms. Carcinogenesis 28: 1104-1110, 2007.

3. Wang X, Lu H, Urvalek AM, et al: KLF8 promotes human breast cancer cell invasion and metastasis by transcriptional activation of MMP9. Oncogene 30: 1901-1911, 2011.

4. Yan C and Boyd DD: Regulation of matrix metalloproteinase gene expression. J Cell Physiol 211: 19-26, 2007.

5. Nagase $\mathrm{H}$ and Woessner JF Jr: Matrix metalloproteinases. J Biol Chem 274: 21491-21494, 1999.

6. Egeblad M and Werb Z: New functions for the matrix metalloproteinases in cancer progression. Nat Rev Cancer 2: 161-174, 2002.

7. Kessenbrock K, Plaks V and Werb Z: Matrix metalloproteinases: regulators of the tumor microenvironment. Cell 141: 52-67, 2010.

8. Chambers AF and Matrisian LM: Changing views of the role of matrix metalloproteinases in metastasis. J Natl Cancer Inst 89: 1260-1270, 1997.

9. Scorilas A, Karameris A, Arnogiannaki N, et al: Overexpression of matrix-metalloproteinase-9 in human breast cancer: a potential favourable indicator in node-negative patients. Br J Cancer 84: 1488-1496, 2001.

10. Nabeshima K, Inoue $T$, Shimao $Y$ and Sameshima T: Matrix metalloproteinases in tumor invasion: role for cell migration. Pathol Int 52: 255-264, 2002.

11. Bailly C: Topoisomerase I poisons and suppressors as anticancer drugs. Curr Med Chem 7: 39-58, 2000.

12. Masuda Y, Nishida A, Hori K, et al: $\beta$-hydroxyisovaleryl shikonin induces apoptosis in human leukemia cells by inhibiting the activity of a polo-like kinase 1 (PLK1). Oncogene 22: 1012-1023, 2003.
13. Kim SH, Kang IC, Yoon TJ, et al: Antitumor activities of a newly synthesized shikonin derivative, 2-hyim-DMNQ-S-33. Cancer Lett 172: 171-175, 2001.

14. Masuda Y, Shima G, Aiuchi T, et al: Involvement of tumor necrosis factor receptor-associated protein 1 (TRAP1) in apoptosis induced by $\beta$-hydroxyisovalerylshikonin. J Biol Chem 279: 42503-42515, 2004.

15. Yoon Y, Kim YO, Lim NY, Jeon WK and Sung HJ: Shikonin, an ingredient of Lithospermum erythrorhizon induced apoptosis in HL60 human premyelocytic leukemia cell line. Planta Med 65: $532-535,1999$.

16. Yang $\mathrm{H}$, Zhou $\mathrm{P}$, Huang $\mathrm{H}$, et al: Shikonin exerts antitumor activity via proteasome inhibition and cell death induction in vitro and in vivo. Int J Cancer 124: 2450-2459, 2009.

17. Gaddipati JP, Mani H, Shefali, et al: Inhibition of growth and regulation of IGFs and VEGF in human prostate cancer cell lines by shikonin analogue 93/637 (SA). Anticancer Res 20: 2547-2552, 2000.

18. Wu Z, Wu L, Li L, Tashiro S, Onodera S and Ikejima T: p53-mediated cell cycle arrest and apoptosis induced by shikonin via a caspase-9-dependent mechanism in human malignant melanoma A375-S2 cells. J Pharmacol Sci 94: 166-176, 2004.

19. Chung TW, Moon SK, Chang YC, et al: Novel and therapeutic effect of caffeic acid and caffeic acid phenyl ester on hepatocarcinoma cells: complete regression of hepatoma growth and metastasis by dual mechanism. FASEB J 18: 1670-1681, 2004.

20. Hu B, Thirtamara-Rajamani KK, Sim H and Viapiano MS: Fibulin-3 is uniquely upregulated in malignant gliomas and promotes tumor cell motility and invasion. Mol Cancer Res 7: 1756-1770, 2009

21. Huang W, Yu LF, Zhong J, et al: Angiotensin II type 1 receptor expression in human gastric cancer and induces MMP2 and MMP9 expression in MKN-28 cells. Dig Dis Sci 53: 163-168, 2008.

22. Jin H, Pan Y, He L, et al: p75 neurotrophin receptor inhibits invasion and metastasis of gastric cancer. Mol Cancer Res 5: 423-433, 2007.

23. Min R, Zun Z, Min Y, Wenhu D, Wenjun Y and Chenping Z: Shikonin inhibits tumor invasion via down-regulation of NF-kBmediated MMP-9 expression in human ACC-M cells. Oral Dis 17: 362-369, 2011.

24. Sato T, Koike L, Miyata Y, et al: Inhibition of activator protein-1 binding activity and phosphatidylinositol 3-kinase pathway by nobiletin, a polymethoxy flavonoid, results in augmentation of tissue inhibitor of metalloproteinases-1 production and suppression of production of matrix metalloproteinases-1 and -9 in human fibrosarcoma HT-1080 cells. Cancer Res 62: 1025-1029, 2002.

25. Gum R, Lengyel E, Juarez J, et al: Stimulation of 92-kDa gelatinase B promoter activity by ras is mitogen-activated protein kinase kinase 1-independent and requires multiple transcription factor binding sites including closely spaced PEA3/ets and AP-1 sequences. J Biol Chem 271: 10672-10680, 1996.

26. Yokoo T and Kitamura M: Dual regulation of IL-1 beta-mediated matrix metalloproteinase- 9 expression in mesangial cells by NF-kappa B and AP-1. Am J Physiol 270: F123-F130, 1996.

27. Matthews CP, Colburn NH and Young MR: AP-1 a target for cancer prevention. Curr Cancer Drug Targets 7: 317-324, 2007.

28. Milde-Langosch K: The Fos family of transcription factors and their role in tumourigenesis. Eur J Cancer 41: 2449-2461, 2005.

29. Ozanne BW, Spence HJ, McGarry LC and Hennigan RF: Transcription factors control invasion: AP-1 the first among equals. Oncogene 26: 1-10, 2007. 